\title{
Caractérisation des Personnages en Théatre Sartrien Vis-À-Vis de L'idéologie Sartrienne
}

\author{
Chioma Faith Uzoho* \\ DOI: http://dx.doi.org/10.4314/ujah.v12i2.11
}

\section{Résumé}

Généralement, on considère Jean-Paul Sartre comme le symbole de la littérature française $d u 20^{e}$ siècle particulièrement en ce qui concerne la littérature engagée. Toutes ses œuvres se portent sur les problèmes de l'existence humaine dont il était question tout au long du $20^{e}$ siècle et même jusqu'à nos jours. On note que la plupart d'ouvrages critiques sur Sartre sont surtout sur ses cuvres théoriques et romanesques; ses ouvres théâtrales n'ont pas été assez. abordées. Dans cette communication, il nous convient de concentrer sur son ouvre théâtrale. D'ailleurs, le théâtre pour Sartre est comme un 'laboratoire' pour l'expérimentation de ses idées philosophiques et politiques basant surtout sur l'existentialisme athée. On constate que chez Sartre, il s'agit essentiellement de théâtre d'idéologie qui a le mérite d'illustrer et de mettre en scène, la quasitotalité des thèmes sartriens déjà soulevés dans ses æeuvres théoriques et romanesques. Nous nous appuierons surtout sur trois ouvres théâtrales de Sartre, à savoir, Huis clos, Les mouches et Les mains sales puisque les trois pièces font bien ressortir l'objectif de notre étude, à savoir, la conformité ou la non-conformité du héros sartrien par rapport à l'idéologie existentialiste sartrienne.

\section{Abstract}

Generally speaking, Jean-Paul Sartre is considered to be the symbol of 20th Century French literature particularly as 
regards committed literature. All his works are based on the problems of human existence which was in question all through the 20th Century and still remains so even in our days. We note that most of the critical works on Sartre are on his theoretical and romantic works; not much has been done on his dramatic works. In this article, we intend to concentrate on his dramatic works. Moreover, drama, for Sartre, can be likened to a 'laboratory' for the experimentation of his philosophical and political ideas based mainly on atheist existentialism. We observe that Sartre represents more of a theater of ideas which has the advantage of illustrating and representing most of the sartrean themes already raised in his theoretical and romantic works. We shall focus mainly on three dramatic works of Sartre, namely, Huis clos, Les mouches, and Les mains sales since the three plays clearly portray the objective of our study, namely, the conformity or the non-conformity of the sartrean hero to the sartrean existentialist ideology.

\section{Introduction}

Le terme 'personnage' est souvent associé avec l'œuvre de fiction ou tout simplement, les auvres littéraires. Il s'agit d'un être imaginaire qui figure dans une œuvre littéraire et qui doit être incarné par un acteur ou une actrice. Quand on parle de la caractérisation, il s'agit d'un procédé de la création et du développement des personnages. Ceci consiste aussi à faire une construction détaillée des personnages. Il s'agit aussi d'une définition précise de l'identité du personnage, particulièrement en ce qui concerne sa psychologie.

Le personnage principal d'une œuvre littéraire est très important parce que la plupart du temps, c'est à travers lui que le lecteur ou le spectateur selon le cas suit l'intrigue ou 
l'histoire de l'œuvre. Il est parmi les autres personnages, le plus important parce que c'est lui qui démontre par son comportement et ses rôles, l'idéologie et la pensée de l'auteur. Ainsi, c'est lui qui porte la plupart du temps, le message de l'auteur.

Nous comptons examiner ici la représentation de la généralité des personnages en théâtre sartrien à la lumière de l'idéologie sartrienne. Dans quelle mesure est-ce que les personnages du théâtre sartrien arrivent à s'intégrer à l'idéologie sartrienne? Les œuvres théâtrales de Sartre sont plus ou moins les représentations des idées soulevées dans ses ouvrages théoriques, c'est-à-dire que ses ouvrages théâtraux sont inspirés par les théories philosophiques qu'il a présentées à travers ses œuvres théoriques. On suppose alors que les comportements de ses personnages en théâtre sont des démonstrations de la relation étroite qui existe entre ses œuvres théoriques et ses œuvres théâtrales. Ce qui reste, c'est de voir si le dramaturge présente tous ces personnages de la même façon sans tenir compte de leurs situations et personnalités particulières. Autrement dit, sont-ils tous de vrais représentants de l'existentialisme athée de Sartre ? Nous allons ainsi tenter une analyse critique des comportements et de la psychologie des personnages principaux des pièces choisies. Il s'agit ainsi d'un essai de marier les comportements de ces personnages à l'idéologie existentialiste de Sartre. Cela nous permettra de découvrir de tout près, combien ses personnages sont de vrais représentants de la généralité des idéologies philosophiques sartriennes.

\section{Caractérisation des personnages en Théâtre Sartrien}

Les pièces sartriennes mettent en lumière d'une manière profonde, le thème de la responsabilité humaine face au destin tout en nous permettant d'étudier directement la 
pensée sartrienne dont le thème central est la liberté. La lecture du drame sartrien est ainsi la meilleure manière d'étudier ses idées. Par exemple, Les mouches reprend le thème de la liberté, celle d'une conscience individuelle. En ce sens, cette pièce est au théâtre, ce que La nausée est au roman. De même, Huis clos est symétrique du Mur (1939): il $\mathrm{y}$ a dans les deux ouvrages, une réflexion du thème de la mauvaise foi.

Les pièces sartriennes présentent des situations réelles ainsi que des manières différentes d'application de la théorie philosophique à une réalité concrète. On y retrouve diverses illustrations des concepts de la liberté et de la responsabilité de l'homme telles que les conçoit Sartre. Ceci est rendu possible par la pluralité des voix qu'il fait raisonner dans les pièces. Le théâtre sartrien nous sert à découvrir des situations, si générales qu'elles soient communes à tous, à partir desquelles on peut proposer aux spectateurs, plusieurs idées que représentent les personnages. Pour analyser les actions de ses personnages et leurs conséquences, il faut placer les personnages dans des situations diverses qui mettent en jeu leur liberté. La liberté se définit alors par le choix que fait l'homme dans une situation donnée de même que par le besoin qu'il ressent d'assumer les conséquences de ses actes. C'est cette situation dans laquelle le personnage est placé qui met en scène de manière concrète le thème central de l'idéologie sartrienne, à savoir, la liberté.

Pour nous, le genre théâtral possède des atouts qui justifient sa préférence au genre romanesque: parmi ces atouts, on peut compter l'intensité de son impact sur les spectateurs ainsi que les leçons morales et urgentes que produit la dramatisation. Les pièces choisies font partie de ce que Sartre appelle 'théâtre de situations' où les actes des personnages sont interprétés à la lumière de leur contexte puisque le contexte explique mieux l'existence humaine que 
la psychologie et les caractères. C'est-à-dire, que les réflexions viennent surtout des situations dans lesquelles se trouvent les personnages. Ceci s'oppose à la tradition du théâtre de caractère. Sartre affirme à ce propos dans son Théâtre de situation :

L'homme n'a pas un caractère fixe, une nature
prévisible. Il n'est rien de déterminé, mais un
devenir qui se détermine et se transforme par des
choix successifs dans des situations.
Le ressort de l'écriture n'est donc plus
"psychologique » il Est dans l'action choisie
librement face à des situations limitées(12).

Pour Sartre, l'homme n'a pas de nature prévisible. Il est donc un devenir qui se détermine et se transforme par des choix successifs selon les situations. Étant donné ce fait que l'homme est un devenir, ce qui compte pour Sartre, c'est de voir l'attitude adoptée par un personnage dans des situations différentes et non pas nécessairement la manifestation de sa psychologie ou d'un caractère inné puisque la nature humaine est inexistante. Sartre explique par là que le but principal du théâtre de situation est d'explorer toutes les situations qui sont les plus communes à l'expérience humaine. Ainsi, on verra des personnages qui diffèrent les uns des autres dans des situations différentes.

Les mains sales est une pièce qui met en scène les convictions marxistes et socialistes de Jean-Paul Sartre. On la voit parfois comme la pièce la plus populaire des pièces sartriennes. Il est évident aussi que tout comme les autres pièces sartriennes, c'est une pièce qui enrichit le pilier philosophique qui la soutient. Sartre présente son personnage principal, Hugo comme celui qui se définit par un acte 'authentique' qui est pour Sartre, une démonstration vive de 
la liberté et de la responsabilité. Son crime est authentique dans la mesure où il choisit lui-même la proposition que lui fait le parti d'assassiner Hoederer. Il décide de le faire et de revendiquer l'acte en se déclarant 'non récupérable' (245). L'explication que Hugo donne à propos de son meurtre fait penser à L'Etranger de Camus où Meursault n'arrive pas à expliquer le mobile de son crime. Hugo déclare que :

Le hasard a tiré trois coups de feu, comme dans les mauvais romans policiers ... Je n'arrive pas à séparer le meurtre de ses motifs ... Tiens, je peux me dire ... que j'ai tué par passion politique et que la fureur qui m'a pris, quand j'ai ouvert la porte, n'était que la petite secousse qui m'a facilité l'exécution'(232).

A ce moment, Hugo ne se définit pas en fonction de son acte parce qu'il ne peut pas affirmer que c'était un acte volontaire. Son premier acte de volonté viendra plus tard lorsqu'il décide de s'éloigner de la nouvelle idée politique du parti après la mort d'Hoederer. On voit un autre point de ressemblance entre Hugo et Meursault quand Hugo dit à Olga concernant son acte:

... un acte, ça va trop vite. Il sort de toi brusquement et tu ne sais pas si c'est parce que tu l'as voulu ou parce que tu n'as pas pu le retenir ... Ce n'est pas moi qui ai tué, c'est le hasard. Si j'avais ouvert la porte deux minutes plus tôt, ou deux minutes plus tard, je ne les aurais pas surpris dans les bras l'un de l'autre, je n'aurais pas tiré ... (232). 
Meursault et Hugo semblent avoir accompli leurs actes sans s'assurer de l'authenticité de l'acte. Les deux actes seront plus tard qualifiés d'authentiques quand les criminels décident de ne pas renoncer aux actes. Auparavant, chacun semble avoir accompli un acte sans qu'il ait été pleinement voulu par sa conscience.

Comme nous l'avons déjà signalé, le théâtre sartrien est un théâtre d'idéologie qui a le mérite d'illustrer et de mettre en scène, presque tous les thèmes déjà soulevés dans ses œuvres romanesques. Comme le remarque Lecherbonnier, 'les ... pièces de Sartre forment un ensemble qui permet de suivre pratiquement la formation de l'idéologie sartrienne' (63). Sartre lui-même signale dans son Théâtre de situations que :

S'il est vrai que l'homme est libre dans une situation donnée ... et qu'il se choisit lui-même dans cette situation, alors il faut montrer au théâtre des situations simples et humaines et des libertés qui se choisissent dans ces situations ... Ce que le théâtre peut montrer de plus émouvant est un caractère en train de se faire, le moment du choix, de la libre décision qui engage une morale et toute une vie (26).

Bien que la liberté humaine pour Sartre soit toujours liée à la situation dans laquelle l'individu se trouve, la recherche de cette liberté chez le héros sartrien est souvent problématique et pleine de difficultés. C'est qu'il existe des pièges tout au long du chemin. Ces pièges se présentent parfois sous formes des situations changeantes ou des desseins des autres. Il y a l'exemple d'Electre qui n'arrive pas à se réaliser à cause de la présence et du jugement de Jupiter. 
Ainsi, on peut dire que chez Sartre, la liberté est une affaire non seulement individuelle mais aussi personnalisée.

Sartre fait à travers Huis clos une illustration du fait que l'homme est beaucoup influencé par le regard (jugement) d'un autre. La pièce est tout à fait différente des autres pièces sartriennes dans la mesure où la portée de la pièce est entièrement philosophique. Il y a aussi le fait que les personnages sont tous des morts. De plus, c'est une pièce qui occupe une place bien déterminée, celle de la mauvaise foi que Sartre a combattue toute sa vie. Huis clos représente une analyse psychologique du comportement des êtres humains en relation avec autrui. Ses thèmes principaux, à savoir, la mauvaise foi et le regard d'autrui sont tirés de la théorie sartrienne énoncée dans L'Etre et le néant où il expose les problèmes relatifs au rapport entre les humains ainsi que la différence entre un homme et un objet. Cette différence réside dans le fait que le premier a une conscience, ce que l'objet ne possède pas. L'objet ne pense pas, il est ainsi enfermé en lui-même (l'en-soi). Par opposition à l'objet, l'homme est capable de réfléchir ; par sa pensée, il peut juger le monde. Il vit ainsi pour soi-même (le pour-soi) puisqu'il est capable de se déterminer. Cette qualité exige chez l'homme un devoir très essentiel: celui d'assumer les responsabilités de son choix et du jugement qu'il porte sur le monde.

La responsabilité exigée chez l'homme à l'exécution de sa liberté par le moyen d'actions volontaires entrâne souvent la peur, l'angoisse et même les incertitudes, ce qui fait que certains êtres préfèrent ignorer leur liberté en s'abandonnant aux autres qu'ils laissent choisir pour eux tandis qu'ils se penchent sur les jugements de ces derniers. Cela, d'après Sartre, aboutit à la mauvaise foi, c'est-à-dire, l'incapacité d'assumer sa responsabilité. C'est le cas des personnages de Huis clos qui sont condamnés à l'enfer 
sartrien pour le fait qu'ils n'ont pas pu assumer la liberté que leur imposait leur situation d'hommes. Puisqu'ils étaient libres comme tout homme sur terre, ils devaient conduire eux-mêmes leur existence au lieu de se laisser influencer par les autres. Cela explique pourquoi Garcin ne peut pas quitter la salle de torture même après avoir aperçu que la porte n'est plus fermée. Il ne peut pas supporter la responsabilité d'affronter sa décision de fuir. Il s'abandonne ainsi à Inès qui doit le juger et définir pour lui son essence. De même, Estelle n'est pas sure qu'elle existe; elle a besoin de se contempler devant un miroir pour se voir comme les autres la voient. Tout comme Garcin, elle doit passer par le regard d'Inès pour se connaître. Quand enfin Inès lui dit qu'elle a des boutons sur le visage, elle accepte ce jugement de l'autre à cause de sa mauvaise foi.

On dirait que cette attitude de se soucier du 'regard d'autrui' chez les personnages de Huis clos les rend en quelque sorte, inauthentiques par rapport à l'idéologie sartrienne. Cela donne l'impression que leur essence est créée par Inès. On retrouve la même situation chez les Argiens dans Les mouches dont l'essence est créée par Jupiter et Egisthe. Ils sont obligés de se soumettre chaque année à une cérémonie de remords. On entend leurs confessions :

Une jeune femme Eh bien oui, je l'avoue, je l'ai trompé tant que j'ai pu ... il souffre ... La foule Egisthe ! Egisthe ! Pitié ! Une femme ! Ah ! Oui ! Pitié ! Pitié ! Personne n'aura donc pitié de moi ! Un homme Je pue ! Je pue ! Je suis une charogne immonde. Voyez, les mouches sont sur moi ... Les femmes Pitié ! Nous sommes entourées de vos visages ... Nous portons votre deuil éternellement et nous pleurons de l'aube à la nuit et de la nuit à l'aube ... Les hommes Pardonnez- 
nous de vivre alors que vous êtes morts. Les enfants Pitié ! Nous n'avons pas fait exprès de naitre, et nous avons honte de grandir. Comment aurions-nous pu vous offenser ?... Les hommes Pardonnez-nous de vivre alors que vous êtes morts. (151-158).

Par leur soumission à la vie du repentir (pour le meurtre commis par le roi Egisthe), les Argiens ne se reconnaissent pas comme des êtres libres qui ne doivent pas se soumettre à une autorité quelconque. A travers ce 'meaculpisme', Sartre fait une caricature des chrétiens qui se donnent à la vie du repentir tout en les voyant comme ignorants. Il satirise aussi la notion de Dieu quand il fait dire Egisthe que les dieux sont injustes (196).

On observe pourtant que la nature de la captivité dans Les mouches diffère de celle de Huis clos dans ce sens que les captifs des Mouches sont à la merci des morts qui les jugent, mais dans Huis clos, les morts n'ont aucune portée sur le monde des vivants. Ce sont plutôt les vivants qui les jugent; les morts sont ainsi à la merci des vivants qui les jugent sans cesse. Les trois personnages voient ce qui se passe sur terre, ils s'inquiètent du jugement que les vivants portent sur eux. Garcin voudrait prêter toute son attention à ceux qui parlent de lui :

Je vous dis de me laisser. Il y a quelqu'un qui parle de moi au journal et je voudrais écouter ... Je ne vous demandais rien, rien que la paix et un peu de silence. Gomez parlait, debout entre les tables, tous les copains du journal écoutaient ... Je voulais comprendre ce qu'ils disaient, c'était difficile : les événements de la terre passent si vite. Est-ce que vous ne pouviez pas vous taire? 
A présent, c'est fini, il ne parle plus, ce qu'il pense de moi est rentré dans la tête ... (52).

Garcin et Estelle n'arrivent même pas à oublier leur passé qui les tourmentent sans cesse. Bien qu'ils soient morts, ils 'regardent' toujours leurs amis qui sont dans le monde et essaient d'imaginer comment ils les jugent. Ils croient que c'est en gardant le souvenir du passé qu'ils peuvent justifier leur existence. Garcin explique même que son destin dépend du jugement des autres. Puisque l'homme n'est pas un être isolé, il se trouve toujours en rapport avec les autres; il est ainsi normal que sa pensée tienne compte de celle des autres puisque la liberté d'un autre peut supprimer celle de l'autre.

D'après Sartre, l'existence et la liberté de l'un constituent un danger pour l'autre car, dit-il dans L'Etre et le néant, 'l'autre me juge, me pense, fait de moi l'objet de sa pensée. Je dépends de lui, sa liberté (son pour-soi) m'a réduit à l'état d'objet (d'en-soi)' (326). Ce point de vue est démontré tout au long de Huis clos où les personnages se jugent. Chacun est à la fois bourreau et victime des deux autres. Celui qui est jugé devient chez son juge un objet. Ils jugent les uns les autres et deviennent ainsi sujets et objets. Le sujet est celui qui juge tandis que l'objet est celui qui est jugé. Jeanson voit ce fait comme 'le problème central de Huis clos' (215). C'est ainsi que les rapports entre les consciences humaines entraînent un conflit.

Tremblay, dans son article en ligne sur Huis clos décrit la pièce comme 'une pièce de théâtre à conflits, à situations conflictuelles'. Les personnages malgré leur condition essayent quand même de se supporter 'mutuellement' comme les personnages de Beckett dans En attendant Godot. Mais lorsque Garcin et Estelle veulent s'aimer, Inès survient : 
Faites ce que vous voudrez, vous êtes les plus forts. Mais rappelez-vous, je suis là et je vous regarde. Je ne vous quitterai pas des yeux, Garcin; il faudra que vous l'embrassiez sous mon regard. Comme je vous hais tous les deux ! Aimez-vous, aimez-vous! Nous sommes en enfer et j'aurai mon tour (74-75).

Rien ne marche parce que sous son regard, les deux amants seront réduits aux objets, car, comme le disait Sartre dans L'Etre et le néant, 'il suffit que les amants soient 'regardés' ensemble par un tiers pour que chacun éprouve l'objectivation (car)...l'amour est un absolu perpétuellement relativisé par les autres' (444). Parmi les personnages de Huis clos, seul Inès tente à affronter sa responsabilité et son angoisse, ce qui sera une étape à l'affirmation de sa liberté et de son existence selon l'idéologie sartrienne.

Le personnage sartrien est souvent affronté du dilemme qui oppose son désir de la solitude, c'est-à-dire, sa peur du jugement de l'autrui. L'intrusion de l'autre signifie pour lui, une rupture du cadre de sa liberté humaine. C'est ainsi qu'on entend dire Estelle à Garcin et à Inès qui la dérangent par leur regard dans la scène: 'laissez-moi tranquille. Vous me faites peur. Je veux m'en aller! Je veux m'en aller !'(259) Elle tente de se débarrasser de ces regards vicieux mais elle ne peut pas puisqu'elle est enfermée pour toute éternité avec ceux qui la jugent. Ceci est aussi le cas de François dans Morts sans sépulture lorsque les membres de son groupe essaient de le forcer de se taire contre son désir. Par contre aux autres résistants, il a à la fois peur de la torture et du jugement de ses collègues ; il prétend alors s'identifier avec eux au début mais sera étranglé une fois qu'ils découvrent sa lâcheté. Le même désir de la solitude se voit chez Lizzie (héroïne de La putain respectueuse) et chez 
Electre qui voulaient au début choisir les voies tout à fait différentes des voies communes. Electre, par la révolte et le désir d'une vengeance, refuse la participation dans la cérémonie des morts, Lizzie ne veut pas signer le faux témoignage malgré le fait qu'elle est seule dans cette décision. Certains d'entre les personnages n'arrivent pas à transcender cette situation de dilemme, ce qui veut dire qu'ils ne se montrent pas assez libres pour pouvoir transcender leurs situations. Ces deux personnages féminins sont victimes de la situation décrite.

On voit dans la dernière scène de Huis clos, la vision sartrienne de l'enfer lorsqu'il déclare par la bouche de Garcin : 'vous vous rappelez : le soufre, le bûcher, le gril ... Ah! Quelle plaisanterie. Pas besoin de gril, l'enfer, c'est les autres' (92). Avant ce moment, les personnages croyaient qu'il arriverait bientôt un bourreau pour les torturer comme à l'enfer. Mais voilà qu'ils découvrent qu'ils sont eux-mêmes les bourreaux et doivent rester ensemble pour pouvoir torturer chacun les autres :

Inès Ha ! Attendez ! J'ai compris pourquoi ils nous ont mis ensemble. Garcin Prenez garde à ce que vous allez dire. Inès Vous allez voir comme c'est bête. Bête comme chou ! Il n'y a pas de torture physique, n'est-pas? Et cependant, nous sommes en enfer. Et personne ne doit venir. Personne ... En somme, il y a quelqu'un qui manque ici : c'est le bourreau ... ils ont réalisé une économie de personnel ... Ce sont les clients qui font le service eux-mêmes, comme dans les restaurants coopératifs ... Le bourreau, c'est chacun de nous pour les deux autres (41). 
Un auteur anonyme d'un article en ligne intitulé 'les visions sataniques de l'au-delà', voit cette vision de l'enfer selon Sartre comme 'l'une des plus sataniques de toutes les définitions de l'enfer'. La phrase de Garcin /Sartre et la citation ci-dessus résument le thème central de la pièce. Pour lui, la présence des autres peut parfois constituer un véritable enfer; le regard d'autrui est à la fois insupportable et tourmentant comme à l'enfer.

Bien qu'ils soient morts, les personnages de Huis clos éprouvent des sentiments et parlent. Leur comportement montre qu'ils nient leur liberté ; ils sont donc de mauvaise foi. Ceci consiste à fuir leur liberté et responsabilité en croyant qu'il est plus facile d'obéir aveuglement aux ordres et aux valeurs préétablis que d'insister sur une voie individuelle pour assumer pleinement la responsabilité de leurs actes authentiques. La mauvaise foi consiste ainsi à donner des arguments et des excuses pour l'ensemble de leurs actions. Tout comme les Argiens dans Les mouches, qui doivent subir une cérémonie du remords chaque année, Garcin et Estelle passent leur temps à vouloir s'excuser leurs actions passées: la désertion et l'infanticide. Ils se construisent des excuses pour éviter la responsabilité de leurs vies d'autrefois.

Contrairement à Garcin qui faisait semblant de ne pas avoir peur dès son entrée dans l'enfer, Inès admet qu'elle a peur et refuse de se faire des illusions comme les deux autres. Elle se caractérise comme une femme méchante. Elle est complètement consciente de ce qu'elle a fait et revendique sa situation. Elle est lesbienne, elle a tué son cousin, le mari de Florence avec laquelle elle a eu une affaire. Elle l'a tué pour pouvoir s'approcher de Florence sans résistance. Elle dit à Estelle :

Nous sommes en enfer, ma petite, ... on ne damne jamais les gens pour rien ... Damné, ... 
Nous avons eu notre heure de plaisir, n'est-ce pas? Il y a des gens qui ont souffert pour nous jusqu'à la mort et cela nous amusait ... A présent, il faut payer (40).

Cette prise de conscience lui permet de voir la mauvaise foi de Garcin et d'Estelle. Elle admet ses fautes et son châtiment, et plus loin : 'Je ne regrette rien, ... moi, je suis méchante : ça veut dire que j'ai besoin de la souffrance des autres pour exister' (57). C'est cette indifférence au passé qui la distingue des deux autres personnages. Pour elle, exister, c'est éprouver le mépris de l'autre et manifester à la fois la méchanceté envers les autres.

A travers La putain respectueuse, on voit une démonstration très nette de la mauvaise foi telle que la conçoit Sartre. Le nègre est en train de s'enfuir tout au long de la pièce ; malgré le fait qu'il n'est pas coupable du crime dont on l'accuse, il n'arrive ni à se défendre ni à affronter les accusateurs. Son rôle tout au long de la pièce, c'est de s'enfuir continuellement parce que les Blancs sont après lui. Même quand Lizzie est prête à le soutenir, il refuse de se défendre et rejette le revolver que lui offre Lizzie pour sa défense : 'Je ne peux pas Madame ... Je ne peux pas tirer sur des blancs' (77).

Huis clos est comme nous l'avons déjà remarqué, une œuvre philosophique à travers laquelle Sartre démontre son refus pour le théâtre réaliste qui, d'après lui, est pessimiste. Il préfère baser plutôt ses thèmes sur la condition humaine tout en présentant à l'homme moderne, une peinture fidèle de luimême, ses problèmes, ses espoirs et ses luttes. Son opinion, c'est que 'Le théâtre doit s'adresser aux masses ... leur parler de leur préoccupations les plus générales, exprimer leurs inquiétudes sous la forme de mythes que chacun puisse 
comprendre et ressentir profondément' (Théâtre de situations, p. 65).

Généralement, les personnages sartriens sont susceptibles au changement brusque de conduite. Par exemple, Oreste abandonne les Argiens pour lesquels il a tué le roi Egisthe. Il déclare à son peuple: ‘ ... Je veux être un roi sans terre et sans sujets. Adieu, mes hommes, tentez de vivre : tout est neuf ici ... pour moi, la vie commence ... une étrange vie' (244). Il quitte à jamais la ville d'Argos poursuivie par les mouches. Par ce changement brusque, on a l'impression qu'il assume le rôle d'un bouc émissaire ou de l'agneau qui prend sur lui les 'péchés' des Argiens en se sacrifiant pour les autres. En décidant le sort des autres, il choisit en même temps sa propre règle de conduite. Néanmoins, son acte a un impact universel et rédempteur parce qu'il le fait pour tous les Argiens. Electre de sa part se donne à la vie du remords au lieu de continuer dans la liberté qu'elle a prétendu démontrer au début de la pièce lorsqu'elle refuse d'assister à la cérémonie du remords en habit de deuil. Elle ne peut plus assumer la responsabilité du meurtre auquel elle a participé.

On voit aussi ce changement brusque chez les membres du Parti Prolétarien de Hugo dans Les mains sales qui adoptent la politique de Hoederer qu'ils avaient rejetée au début. Tel est le cas du personnage principal de La putain respectueuse Lizzie qui change tout à coup d'avis après avoir signé un faux témoignage que le viol dans le train a été commis par le noir. Tout comme Electre, elle avait auparavant, décidé de suivre une voie qui n'était pas celle des autres : celle de choisir elle-même, comment affirmer son existence. Elle est tourmentée par le remords et regrette le fait qu'elle ait signé le témoignage. C'est un fait qui la met aux prises avec sa conscience. On la voit manifester la mauvaise foi, ce qui d'après Sartre, signifie l'incapacité d'assumer la 
responsabilité d'un acte authentique quoique le cas de Lizzie ne soit pas un acte authentique puisqu'elle a été entrainée dans l'affaire par la ruse du Sénateur. Ce changement brusque constitue le problème des résistants de Morts sans sépulture. Ils décident de 'parler' même après avoir juré de mourir dans la torture au lieu de parler.

Canoris Il reste cinq minutes ... Lucie Cinq minutes, oui. Et qu'espères-tu ? Me convaincre en cinq inutes? Canoris Oui. Lucie... Allezvous-en! Allez vivre, puisque vous pouvez accepter. Moi, je ... souhaite qu'après ma mort tout soit sur terre comme si je n'avais jamais existé ... Un milicien Il reste deux minutes ... Canoris Va dire à tes chefs que nous allons parler ... Il faut vivre. va dire à tes chefs que nous allons parler ...sur la route de Grenoble, à la borne 42 , prenez le sentier à main droite. Au bout de cinquante mètres en foret vous trouverez un taillis et derrière le taillis une grotte. Le chef est la avec des armes. (234-239).

Seule Lucie reste stable. Comme Oreste, elle est prête à prendre son acte sur elle: 'j' ai pris tout le mal sur moi ; il faut qu'on me supprime et tout ce mal avec' (234).

Les comportements d'Electre et de Lizzie s'opposent complètement à l'attitude de Lucie dans Morts sans sépulture. Lucie est un personnage sartrien par excellence qui reste le même, prête à assumer la responsabilité de ses actes sans se laisser influencer par des forces extérieures. En ce qui concerne l'attitude d'Oreste, Jeanson est d'avis que cet acte est un acte pour lui seul à travers lequel il se sert des Argiens pour se libérer et se sentir exister. Dans ses mots, 'la liberté d'Oreste est une liberté pour soi et qui considère comme 
inessentielle son existence pour autrui. L'incarnation de la liberté ... demeure ainsi dans la simple intention de s'incarner' (244). Cette opinion se justifie parce qu'Oreste s'éloigne des Argiens malgré le salut qu'il prétend leur avoir apporté. Il abandonne Argos à son sort et garde sa propre indépendance pour continuer son aventure personnelle. Son acte incarne ainsi une libération plus individuelle que collective. Il laisse la cité vide et n'installe aucun nouveau pouvoir. Launay partage cet avis que l'attitude héroïque d'Oreste 'n' a pas abouti à la véritable libération des Argiens parce qu'il quitte la ville ... sans savoir ce qui est arrivé à la pauvre cité après son départ' (111). Michel Contat s'y met d'accord lorsqu'il écrit :

L'acte d'héroïsme solitaire par lequel cette liberté d'Oreste s'affirme est un moyen de salut personnel et non de libération pratique, ... attitude qui correspond à celle de Sartre à une époque où il concevait l'engagement comme un problème de morale individuelle (91).

La liberté d'Oreste peut être considérée comme une liberté paradoxale dans la mesure où elle s'accompagne d'un autre exil; il est poursuivi par les mouches qui autrefois tourmentaient les Argiens. Adrian Hoven, dans son article en ligne intitulé 'Forger des mythes: le théâtre de Sartre, un théâtre de situations' voit Oreste comme celui qui 'échoue nécessairement dans son rôle du nouveau Messie' (n.pag). On note d'ailleurs que la notion de la liberté dans Les mouches n'est pas exactement la même que celle de Huis clos. La différence consiste en ce fait que tandis que Les mouches montre l'épreuve d'une liberté qui va s'accomplir à travers un acte réservé pour le futur, Huis clos propose une liberté qui s'est déjà accomplie. Oreste est en Argos pour réaliser la 
vengeance de son père assassiné par Egisthe en collaboration avec Clytemnestre. Il s'agit alors d'un acte qui n'est pas encore accompli. Les personnages de Huis clos, puisqu'ils sont déjà morts, doivent rendre compte de l'usage de leur 'liberté' lorsqu'ils étaient vivants. De ce fait, Noudelmann remarque que 'dans un cas, la liberté se conjugue au futur, dans l'autre, au passé' (54).

Par contre au théâtre réaliste, Sartre nous présente à travers Les mouches, un théâtre philosophique et politique. Contat et Rybalka soulignent que 'les pièces de Sartre ont été conçues par leur auteur moins en vue d'expériences scéniques ... qu'en fonction d'un projet philosophique' (10). Ceci justifie la nature philosophique de la pièce. On voit à travers l'évolution de ses personnages, une réflexion des étapes nécessaires à l'accomplissement de la liberté humaine que prêche Sartre. Au commencement, Oreste a du mal à s'attacher à la notion conventionnelle de la liberté individuelle, ce qui donne souvent l'impression que l'homme est libre seulement quand il n'est pas alourdi par quelques engagements et responsabilités. Bien que cette notion de liberté soit universellement acceptable, ce n'est pas ainsi que Sartre conçoit la liberté. Celle qu'il nous présente dans Les mouches est basée sur le fait qu'Oreste n'est pas obligé de se soumettre à une autorité hors de lui-même. Il est ainsi libre de ne pas respecter les règles générales, qu'elles soient politiques ou religieuses. Au fur et à mesure que l'action se développe dans la pièce, on voit un Oreste qui a une autre conception de la liberté, à savoir, celle qui devient entrelacée avec l'action; il comprend qu'il doit passer de sa passivité à l'activité en tuant les tyrans ; ce qui doit démontrer sa liberté absolue. Il se crée ainsi une idée nouvelle. Le résultat, c'est qu'il ne ressent pas de remords après le meurtre. Il est à la fois libre d'agir et d'interpréter son action : 
Je suis libre Electre; j'ai fait mon acte et cet acte était bon. Je le porterai sur mes épaules comme un passeur d'eau porte les voyageurs, ... Je suis libre par delà l'angoisse et les souvenirs ... Je ne suis pas coupable et tu ne saurais me faire expier ce que je ne reconnais pas pour un crime (208).

Oreste devient ainsi son propre juge puisque d'après Sartre, les notions du mal et du bien sont subjectives. Il se voit plutôt comme un sauveur (celui qui délivre les Argiens des mains des tyrans) qu'un meurtrier (qui est là pour venger son père).

Par contre à Oreste, Jupiter est le symbole de l'autorité divine. Il symbolise aussi l'image que les gens gardent toujours à l'intérieur d'eux-mêmes, celle qui les fait conscients de l'existence d'un Etre/Juge auquel appartient toute autorité. C'est une opinion qui d'après la doctrine sartrienne, empêche la liberté humaine.

Le personnage de Jupiter représente celui qui joue un tour aux peuples. Il fait mouvoir une pierre sous la caverne pour donner l'impression que les morts sont présents pendant la cérémonie des morts. Il se cache pour écouter la conversation entre Oreste et Electre lorsque ces derniers complotaient le meurtre du roi et de la reine pour ensuite tout révéler à Egisthe comme prophétie. Sartre cherche à démontrer par cette action que toute domination morale ou divine n'est qu'une déception. Egisthe, de sa part, est présenté comme un agent de Jupiter parmi les hommes. Il cherche à imposer l'ordre chez les hommes. Sa méthode consiste à donner aux Argiens l'impression qu'ils sont coupables du meurtre d'Agamemnon et doivent ainsi partager avec lui, le remords en se soumettant chaque année à une cérémonie du remords en s'habillant en noir. Dans ses mots à Jupiter, 'C'est pour l'ordre que j'ai séduit Clytemnestre ... je 
voulais que l'ordre règne et qu'il règne par moi ... j'ai fait de l'ordre' (200). Seuls lui et Jupiter savent que les hommes sont libres et à ce propos, Jupiter remarque dans sa conversation avec Egisthe que 'nous sommes pareils' (199). Cela veut dire qu'ils savent ce que les autres ne savent pas :

Jupiter Nous faisons tous les deux régner l'ordre, toi, dans Argos, moi dans le monde ... le secret douloureux des dieux et des rois : c'est que les hommes sont libres ... Tu le sais, et ils ne le savent pas. Egisthe Parbleu, s'ils le savaient, ils mettraient le feu aux quatre coins de mon palais. Voilà quinze ans que je joue la comédie pour leur mas quer le pouvoir (198).

Egisthe fait régner l'ordre pour qu'il continue à garder le pouvoir. Pour lui, c'est une manière de rendre les Argiens ignorants de leur liberté naturelle. Tout semble ainsi paisible dans la ville puisqu'on a peur du jugement d'autrui et de Jupiter. Les Argiens sont appris qu'ils sont coupables et doivent passer leurs vies dans le repentir. Oreste se forme une conception entièrement différente de la liberté: on est coupable seulement quand on refuse la responsabilité pour son action et se repent. Autrement dit, il est question de la culpabilité seulement quand un individu croit que son action est mauvaise en se jugeant moralement. Pour Oreste un individu ne doit pas ressentir la culpabilité quand il exerce sa liberté car c'est l'homme qui crée sa valeur et son jugement. Sa liberté tout comme celle de Goetz (personnage principal du Diable et le bon dieu) est blasphématoire.

Le héros sartrien est rebelle à l'autorité divine; il bouleverse tout ordre conventionnel. Oreste a été créé libre mais sa liberté s'est tournée contre son 'Créateur'. Il dit à Jupiter : 
Il se peut (que tu m'aies créé libre) mais elle s'est retournée contre toi et nous n'y pouvons rien, ni l'un ni l'autre ... Je ne suis ni le maitre ni l'esclave, Jupiter, je suis ma liberté ! A peine m'as-tu créé que j'ai cessé de t'appartenir (223).

On voit dans cette remarque d'Oreste, une contradiction de l'athéisme sartrien. Pour Sartre, il n'existe aucun pouvoir divin; l'homme surgit dans le monde, se rencontre et se définit après. Il est là et c'est à lui de se définir en entreprenant un acte authentique tout en assumant la responsabilité de cet acte. Mais Oreste qui doit être l'incarnation de l'idéologie sartrienne reconnait le dieu Jupiter comme son créateur. L'évolution d'Electre dans la pièce s'oppose beaucoup à celle d'Oreste. Au début, Oreste n'était pas tout à fait convaincu de sa mission; lors de leur première rencontre, Oreste n'a pas l'intention de faire un acte héroïque; il a plutôt envie de fuir avec elle. Electre, de sa part croit qu'elle a un devoir: celui d'assurer la vengeance de leur père; dès qu'il est réalisé, cela donnera un sens à sa vie. Avant le meurtre, elle est convaincue qu'il sera juste de le faire, mais dès que l'acte est réalisé, elle est déchirée par la passion et s'écrie :

\begin{abstract}
Ah! Je l'ai voulu !... il faut que je le veuille encore ... Celui-ci est mort. C'est donc ce que je voulais. Je ne m'en rendais pas compte... Ah! Je ne peux plus supporter ce regard ... Il l'a frappée. C'était notre mère, et il l'a frappée. Voici : mes ennemis sont morts ... j'ai joui de cette mort par avance ... Est-ce que je me suis menti pendant quinze ans ? Ça n'est pas vrai, ... je ne suis pas lâche ! (205).
\end{abstract}


D'après Jeanson, sa raison de vivre a 'cessé d'être la vengeance pour devenir l'espoir de cette vengeance' (16). C'est comme si Electre n'a pas vraiment voulu la mort de ses ennemis, mais plutôt qu'elle aurait préféré rester dans la haine et le rêve d'une vengeance sans jamais l'atteindre.

Sartre nous présente dans le personnage d'Electre, l'impuissance de l'homme à conquérir sa liberté et les conflits dans lesquels se trouve parfois l'homme qui est à la recherche de sa liberté. Ces conflits peuvent se présenter comme le regard de condamnation que les autres portent sur lui, comme le héros de Huis clos qui ne peut pas s'échapper à la condamnation des hommes malgré son effort de se libérer ; il reste ainsi avec ses compagnons dans l'enclos fortement verrouillé pour toujours. Dans le cas de Hugo dans Les mains sales, le conflit vient de la part de ses camarades qui adoptent plus tard la politique de Hoederer pour laquelle on l'avait envoyé le tuer. Mais de la part d'Electre, le conflit se présente comme la peur causée par la présence de Jupiter et du roi Egisthe qui font régner l'ordre.

Electre, dans sa révolte et recherche pour la liberté est en quelque sorte comparable à Antigone dans Antigone de Jean Anouilh dans la mesure où les deux refusent le remords et le repentir (au début pour Electre), mais tandis qu'Antigone est prête à subir la responsabilité de sa révolte, Electre ne peut pas lutter jusqu'à la fin. Elle ne tardera pas à se jeter dans les bras de Jupiter pour échapper à l'horreur que lui inspire le meurtre dont pourtant elle n'avait pas cessé de rêver. Elle manque du courage requis pour soutenir sa conviction d'autrefois. Le meurtre parait alors pour elle, comme un accident ou un acte qui n'est pas prémédité.

Les yeux, les pierres et les mouches évoqués dans Les mouches peuvent être conçus comme des symboles. On peut 
considérer les yeux comme la vision du futur. A l'exception d'Electre (au début de la pièce) et d'Oreste, les Argiens ont des yeux 'morts' dans la mesure où ils ne voient que leur passé tout comme Garcin et Estelle dans Huis clos. Les yeux symbolisent aussi le regard et le jugement d'autrui. Les pierres sont symboliques de la nature qui représente l'être ensoi, c'est-à-dire, l'objet et toutes choses extérieures à la conscience. De même, le bronze, l'objet lourd et silencieux dans Huis clos représente l'être en-soi dans sa nature entière de passivité. Cela renvoie chaque personnage à son état de mort-vivant. Suivant l'idéologie de Sartre, seule la mort est capable de chosifier le 'pour-soi' (l'homme) parce qu'on devient complètement raide à sa mort. On retrouve les mêmes objets symboliques tels l'aspirateur, le bracelet et le revolver dans d'autres pièces sartriennes, telles, Les mains sales, et La putain respectueuse. Ils sont là, par opposition au 'pour-soi' sans essence, c'est l'homme qui doit déterminer son essence.

Jupiter se présente comme celui qui a le pouvoir de manipuler la nature. Il fait mouvoir les pierres pendant la cérémonie pour prouver qu'on peut exercer son contrôle sur les objets et non pas sur les êtres parce qu'ils sont libres. Ainsi, l'existence de l'homme diffère de celle des objets. L'homme se fait réduire à une 'pierre' (objet) quand il décide de se soumettre à une autorité autre que lui-même. Les mouches représentent le châtiment des dieux à cause du meurtre d'Agamemnon. Elles symbolisent aussi la domination ; elles sont là pour entraîner le repentir chez les Argiens.

Selon Sartre, être libre ne veut pas seulement dire choisir contre une espèce d'esclavage, mais il implique aussi le refus de tout ordre existant y compris tout ce qui s'oppose à la liberté. Un homme libre doit ainsi se ficher du monde autour de lui puisqu'il doit sa justification uniquement à luimême. Hugo dans Les mains sales explique à Olga, 'il n'y 
avait plus d'ordre, ... l'ordre est resté en arrière et je m'avançais seul et j'ai tué seul ...' (22). Après son acte, Oreste se déclare juste :

... J'ai fait mon acte, Electre, et cet acte était bon. Je le porterai sur mes épaules comme un passeur d'eau porte les voyageurs, je le ferai passer sur l'autre rive et $\mathrm{j}$ 'en rendrai compte ... car ma liberté, c'est lui (208).

L'acte d'Oreste est la démonstration principale de sa liberté. C'est pourquoi il revendique fièrement son acte même quand Jupiter essaie de le convaincre d'accepter la vie du remords et du repentir :

Jupiter Et toi, quitte ce ton fier : il ne convient guère à un coupable en train d'expier son crime.

Oreste Je ne suis pas coupable, et tu ne saurais me faire expier ce que je ne reconnais pas pour un crime ... Tourmente-moi tant que tu voudras : je ne regrette rien (224).

Cette idée d'Oreste reflète l'idée existentialiste de l'authentification en général. Il s'agit d'un acte 'intentionnel' que l'homme entreprend pour se définir. Comme l'affirme Lafarge, 'it is the voluntary act and the voluntary act alone which is always endowed with the privilege of freedom' (57). Il est d'avis que pour Sartre, la vraie liberté entraîne une action intentionnelle. Launay remarque aussi que 'la liberté ne devient réelle que dans l'action lorsque l'individu prend ses responsabilités' (9). Il s'ensuit que le choix, l'action, la liberté et la responsabilité sont inséparables dans l'existentialisme sartrien. L'acte d'Oreste est comparable à celui de Lucie dans la mesure où les deux, en tant que 
personnages 'libres', assument la responsabilité de leurs actes authentiques tout en refusant la culpabilité et le sentiment car il n'y a pas de passion chez un héros sartrien. On entend Lucie après la mort de son frère :

Tu es mort et mes yeux sont secs; pardonnemoi : je n'ai plus de larmes et la mort n'a plus d'importance. Dehors, ils sont trois cents, couchés dans les herbes, et moi aussi, demain, je serai froide et nue sans même une main pour caresser mes cheveux. Il n'y a rien à regretter, tu sais: la vie non plus n'a pas beaucoup d'importance. Si tu t'es arrêté en route, c'est que tu n'avais pas encore assez de forces. Personne n'a le droit de te blâmer. (200201).

Pour Hugo Barine, ce qui compte c'est de sauver et de protéger en toutes circonstances les intérêts du Parti et rien que le Parti, ce qui est en opposition avec l'idée qu'il avait avant son entrée au Parti. Ainsi dit-il à Olga : 'Je respecte les consignes, mais je me respecte aussi moi-même et je n'obéis pas aux ordres idiots qui sont faits exprès pour me ridiculiser' (84). Cet individualisme voire égocentrisme se manifeste aussi dans certains aspects de son discours que nous évoquons: 'Quand l'armée rouge chassera le régent, nous aurons le pouvoir pour nous seuls' (188).

A la lecture de ces deux discours, nous constatons que Hugo Barine évoque simultanément les pronoms 'je' et 'nous', ce qui nous signale combien de fois Hugo ramène tout à lui. Les discours évoqués ci-dessus contrarient la position ainsi que le désir de Hugo quand il entrait dans le Parti : 'J'ai adhéré à ce Parti le jour où j'ai compris ce que c'était l'injustice sociale...'(49). Il avait cette aspiration de 
provoquer l'avènement d'une société sans classes et sans injustice sociale où chacun serait récompensé en fonction de son mérite. La liberté sartrienne telle qu'elle est exprimée dans ses pièces libère l'individu de toutes formes de dominations qui voudraient décider ou choisir pour l'homme son essence.

La conception de liberté par Hugo est identique à celle de Sartre (au moins celle qu'il préconisait jusqu'en 1945). Il se voit comme étant capable de tout faire seul selon son désir et sans aucune contrainte ni intérieure ni extérieure. Aussi dit-il à Louis qui lui propose son assistance dans le besogne que lui donne le Parti: 'pas besoin de liaison, ni d'espionnage, je ferais l'affaire moi-même' (52). On peut donc dire que pour Hugo, la liberté est avant tout, une affaire individuelle. Par contre, chez Hoederer, la liberté trouve sa meilleure expression lorsqu'elle fonctionne pour la collectivité. Inès de Huis clos n'a pas non plus besoin d'aide. C'est Garcin qui propose qu'ils doivent s'aider l'un l'autre pour surmonter leur situation :

Garcin Est-ce que nous ne pourrions pas essayer de nous aider les uns les autres? Inès Je n'ai pas besoin d'aide. Garcin ... Aucun de nous ne peut se sauver seul; il faut que nous nous perdions ensemble ou que nous nous tirions d'affaire ensemble ... (62-63).

Inès est comme Hugo des Mains sales et Lucie de Morts sans sépulture, un personnage qui veut ramener tout à lui. Ces personnages préfèrent l'individualisme à la collectivité lorsqu'il s'agit de la démonstration de la liberté. Comme Oreste, Hugo commence par la collectivité et se termine par l'individualisme. La liberté pour Hoederer, c'est penser non seulement à soi-même mais aussi et surtout aux 
autres. Il sera qualifié de traître social en dépit du fait qu'il veuille collaborer avec le Parti au pouvoir dans l'intérêt du peuple. Devant l'avancement de l'armée rouge qui se traduit par des destructions massives, Hoederer ne voit qu'une seule issue. Son Parti doit accepter de faire des concessions dans l'intérêt du peuple: accepter les pourparlers organisés par le Régent en vue de collaborer avec la classe bourgeoise. L'avantage pour Hoederer, à travers cet acte, réside en une simple protection du peuple pour lequel on lutte. De même, en mourant, il protège son assassin en mentant, en se déclarant responsable pour un acte qu'il n'a pas commis. Il déclare avant de mourir : 'je couchais avec la petite' (227).

Comme nous l'avons déjà remarqué, la liberté sartrienne présuppose toujours la responsabilité. C'est pourquoi Hugo assume la responsabilité de son crime en se déclarant, 'non récupérable' à la fin de la pièce. Idéaliste et communiste, tout comme son créateur, il se montre angoissé par le fait que les bas peuples souffrent de la misère et de l'oppression. Il se rompt avec sa famille bourgeoise pour entrer au Parti Communiste, 'j'ai quitté ma famille et ma classe le jour où j'ai compris ce que c'était que l'oppression. En aucun cas je n'accepterais de compromis avec elle' (49). Il dit plus tard à Hoederer: 'Je suis dans le Parti pour m'oublier.' (106). Il raconte l'histoire de son enfance lorsqu'on le forçait de prendre de l'huile de morue :

Alors on m'ouvrait la bouche, ... on m'enfonçait la cuiller jusqu' au fond de la gorge ... une drogue pour te donner faim pendant que les autres, dans la rue se seraient vendus pour un bifteck, ...avec leur pancarte : donnez-nous du pain ... J'ai quitté ma maison. Je suis entré au parti et c'était pour entendre la même chanson (92). 
Hugo explique ici que c'est sa haine contre l'oppression qui l'a poussé à s'engager dans une lutte pour la liberté. Il est entré au Parti pour réaliser son désir de supprimer l'oppression et améliorer la condition de vie des hommes. Il cherche aussi à se définir à travers cette lutte pour la liberté. Comme un vrai personnage existentialiste/sartrien, son rêve était d'engager les autres (surtout les bas peuples) dans cette liberté. Dans ses mots, 'si je suis entré au parti, c'est pour que tous les hommes, secrétaires ou non, en aient un jour le droit' (84). C'est pour prouver sa bonne foi qu'il accepte la proposition du parti d'assassiner Hoederer qui faisait une politique de négociation avec les bourgeois, ces mêmes gens qui opprimaient les pauvres. Il voit l'alliance que propose Hoederer comme une mésalliance et une politique de 'salissement' des mains. Il avait aussi l'intention de devenir un héros libre et bien connu à travers ce meurtre.

Les justes de Camus semble répondre aux mêmes questions que Les mains sales: la fin justifie-t-elle toujours les moyens? Faut-il tuer les représentants d'un pouvoir corrompu et ceux qui s'adhérent à l'inégalité sociale pour faire régner la bonne cause? L'acte révolutionnaire est-il permis pour assurer la pureté dans la vie politique ? Dans Les justes, on voit un groupe de terroristes qui veulent lancer une bombe pour tuer le Grand duc Serge qui est la cause de la misère des gens y compris les enfants. Yanek Kalieyev est prêt à lancer la bombe mais il ne peut pas parce que les enfants sont chez le duc. Cela amène une division parmi les membres du groupe parce qu'il y en a qui sont d'opinion que les enfants aussi peuvent se sacrifier pour la cause de la révolution désirée. Son but réalisé enfin, Yanek sera jeté en prison. La femme du feu duc lui propose un compromis : s'il donne les noms de ses copains, il sera libéré de la prison. Il 
accepte plutôt de mourir comme Hugo des Mains sales. Il sera alors pendu publiquement.

Certains critiques de Sartre comme Chiari et Jeanson croient que Hugo n'a pas vraiment réalisé cet objectif de devenir un héros puisque le parti a adopté la politique de Hoederer après sa mort. C'est lui, Hoederer qui devient à titre posthume le grand héros du parti. Jeanson est d'avis que 'le cri de non récupérable de Hugo est un suicide et un échec total ; en se livrant à la mort, il ne sauve rien du tout' (47). Dussane remarque aussi que 'Hugo a tué pour rien ... son acte n'a plus de sens, ni plus d'existence' (158). L'attitude de Hoederer (comme celle de tous les autres personnages typiquement sartriens) envers la liberté est machiavélique dans la mesure où il veut le pouvoir à tout prix, même en se 'salissant' les mains et en mentant aux camarades. Il dit à Hugo :

Qu'est-ce que tu veux faire du Parti ... ? A quoi ça sert-il de fourbir un couteau tous les jours si l'on n'en use jamais pour trancher? Un parti, ce n'est jamais qu'un moyen. Il n'y a qu'un seul but : le pouvoir ... Je mentirai quand il faudra ... le mensonge, ce n'est pas moi qui l'ai inventé : il est né dans une société divisée en classes ... Tous les moyens sont bons quand ils sont efficaces (191-193).

Son opinion c'est qu'il faut arriver au pouvoir par n'importe quel moyen possible. Il continue plus tard :

Comme tu tiens à ta pureté, mon petit gars ! Comme tu as peur de te salir les mains. Eh bien, reste pur! A qui cela servira-t-il et pourquoi viens-tu parmi nous? La pureté, c'est une idée 
de fakir et de moine. Vous autres, les intellectuels ... vous en tirez prétexte pour ne rien faire. Ne rien faire, rester immobile, serrer les coudes contre le corps, porter des gants. Moi, j'ai les mains sales ... Je les ai plongées dans la merde et dans le sang ... Est-ce que tu t'imagines qu'on peut gouverner innocemment ? (193-194).

Cette conversation permet à Hugo de préméditer son crime, ce qui va constituer ' 1 'authenticité' de son acte et de sa liberté en tant que personnage sartrien. D'après l'idéologie sartrienne, la vraie liberté entraîne toujours une action intentionnelle. Chez Sorbier et Canoris de Morts sans sépulture, (au commencement de la pièce), 'les moyens n'ont pas d'importance ... il n'y a pas d'enjeu ...' (114). Peu importe les moyens, ce qui compte pour eux, c'est de rester dans la torture au lieu de livrer Jean, leur leader. Chez Lucie aussi, peu importe le moyen, il faut faire taire François par la mort. Il s'agit toujours de cette attitude machiavélique qui souligne les personnages sartriens. Cela explique peut-être pourquoi un grand nombre tuent pour réaliser leurs fins.

On dirait que Hugo poursuit trois fins : la révolution dans le parti, la pureté et le moyen de donner un sens à son existence. Pour réaliser ces fins, il cherche une occasion pour tuer Hoederer à cause de sa politique de négociation. Pour s'évoluer à un vrai héros sartrien, il supprime complètement le sentiment d'amitié pour tirer sur sa victime le jour où il le surprend en train d'embrasser Jessica. Launay est d'avis que 'comme Oreste, Hugo est en quête de son salut personnel ... le mobile du crime ... n'est que la jalousie' (13). En tout cas, le fait que Hugo a prémédité ce crime donne l'impression qu'il a saisi l'occasion pour tirer lorsqu'il voit Jessica avec Hoederer. On peut ainsi déduire que le fait qu'il trouve 
Jessica dans les mains de Hoederer lui a simplement servi d'excuse pour accomplir sa tâche. Pourtant, il n'existe aucun amour entre lui et Jessica parce qu'un personnage typiquement sartrien n'a pas de place pour la passion. Avant le meurtre, il avait dit à Jessica: 'Je ne t'en veux pas et je ne suis pas jaloux, nous ne nous aimons pas' (225).

Hugo peut rejoindre le Parti à une seule condition : renier son acte en acceptant qu'il a tué pour des raisons personnelles. Mais, Hugo comprend que s'il renie son acte, il ne peut plus se présenter comme un héros libre et responsable de son acte, ce qui ferait perdre à son acte la marque d'authenticité. Après tout, c'était par le choix libre qu'il avait accepté la proposition du Parti ; par le choix aussi, il refuse de renoncer à son acte, mais se donne librement à la mort. Hugo est en quelque sorte comparable à Egisthe qui accepte de mourir en dépit de l'avertissement de Jupiter qu'Oreste voulait l'assassiner. Il est aussi comparable à Antigone dans Antigone de Jean Anouilh qui choisit librement la mort au lieu de renoncer à son acte. Elle avait enterré le corps de son frère Polynice malgré l'ordre du roi Créon de le laisser pourrir sur la terre et que quiconque tentera l'enterrer serait lui-même enterré vif. Malgré le conseil de son oncle Créon, Antigone refuse de renoncer à son acte mais se donne librement à la mort devant son oncle, comme Hugo devant ses camarades. Les deux refusent toute lâcheté et mauvaise foi. Chez Lucie aussi, on voit ce refus de la mauvaise foi. Elle ne regrette pas le fait qu'elle a fait tuer son frère François. C'est elle plutôt qui console ses collègues. Pour elle, ce qui compte c'est que le petit est par sa mort, devenu l'un d'eux parce qu'il ne parlera pas. Pour ces personnages typiquement sartriens, la fin justifie le moyen. Il faut toujours assumer la responsabilité d'un acte authentique.

Il y a une grande ressemblance entre les buts et la nature des crimes de Hugo et d'Oreste. Leurs crimes servent 
de démonstration de leur liberté personnelle et de moyen de conférer un sens à leur existence. Tandis qu'Oreste veut engager les Argiens à sa liberté, Hugo, de sa part, veut engager les ouvriers opprimés à la sienne. Hugo est aussi à la recherche de la pureté, de l'honnêteté et de la révolution dans la vie politique. Oreste est à la recherche de la justice et de la vengeance de son père. On note aussi que leurs crimes sont des actes intentionnels, ce que Sartre appelle les actes authentiques. Pour insister sur l'authenticité de leurs actes, les deux refusent le remords et le repentir. Ils prennent plutôt la responsabilité de leurs actes selon l'idéologie sartrienne. Condamné par ses camarades, Hugo disparaît à la mort, tandis qu'Oreste disparaît avec les mouches. En ce qui concerne la réalisation de leurs buts, on dirait que les deux criminels ont réussi seulement à se définir à travers leurs actes. Hugo n'a pas pu amener la révolution dans le parti. Il n'a pas non plus amené la liberté des opprimés. Le parti adopte la même politique pour laquelle il a assassiné Hoederer. Bien qu'Oreste réussisse à venger son père, il quitte la ville à jamais et ne sait plus ce qui arrive aux pauvres Argiens.

Le diable et le bon Dieu est une pièce extrêmement philosophique qui aborde surtout une caricature de la notion de Dieu et la critique de la religion chrétienne. C'est un drame critique où on trouve la description du conflit des consciences. On y retrouve le thème de la liberté absolue qui reste une idée centrale de toutes les pièces sartriennes parmi d'autres.

Pour justifier son athéisme, Sartre nous présente l'histoire d'une femme dont l'enfant meurt de faim. Heinrich le curé n'arrive pas à expliquer à la femme pourquoi Dieu permet une telle chose : 
La femme Je veux que tu m'expliques. Heinrich Je ne peux rien expliquer. La femme Tu ne sais même pas de quoi je parle ... Pourquoi l'enfant est mort. Heinrich Quel enfant? La femme Le mien ... il avait trois ans et il est mort de faim. Heinrich Je suis fatigué ma sœur ...(20).

C'est Nasty qui lui explique enfin que quand les riches font la guerre, ce sont les pauvres qui meurent. La situation est en quelque sorte comparable à celle qu'on retrouve dans $L a$ peste de Camus où les enfants meurent de la peste qui a détruit tant de vies dans la ville d'Oran. Personne n'arrive à expliquer pourquoi une telle chose devrait arriver aux enfants qui n'ont pas péché. Camus démontre par cette histoire, l'absurdité de la vie.

Sartre se moque de l'idée de médiateurs entre l'homme et Dieu. Il s'en moque dans le personnage de Nasty dit prophète, qui dit enfin que tous les hommes sont prophètes ou Dieu n'existe pas. C'est-à-dire, pour Sartre, si vraiment Dieu existe, les hommes doivent avoir de rapport direct avec lui et non pas communiquer avec lui par moyen d'un intermédiaire. Il démontre aussi son athéisme dans le héros, Goetz qui se voue au mal par défi en insistant que si Dieu existe, il doit le punir pour ses crimes. Il justifie son choix de vivre dans le mal pour se définir. Puisque l'homme est libre en situation, il est simplement déterminé par sa situation. Il dit à Heinrich que la trahison est liée à la bâtardise. Tout comme Oreste, il se croit juste car pour Sartre, l'homme est son propre juge. Les notions du mal et du bien ne sont pas absolues. Elles sont plutôt subjectives.

A travers le curé Heinrich et Goetz qui sont rejetés par les peuples à cause du fait qu'ils sont bâtards, Sartre fait démontrer son refus de l'oppression. Les deux personnages sont contraints à rejeter le monde à leur tour pour pouvoir 
affirmer leur existence. L'homme pour Sartre, doit faire quelque chose pour échapper à la solitude et se définir. C'est donc par ressentiment contre les hommes qui ne l'ont pas accepté qu'il souhaite vivre dans l'inhumain. C'est aussi comme il nous démontre sa haine pour l'oppression à travers Hugo qui quitte sa famille bourgeoise dans Les mains sales dès qu'il comprend ce que c'est que l'oppression. Goetz va quitter le mal après pour faire le bien. Dans ses mots, 'je parie donc que je ferai le Bien: c'est encore la meilleure manière d'être seul. J'étais criminel, je me change, je retourne ma veste et je parie d'être un saint' (98). Il va par la suite échouer dans son entreprise de faire du bien. A la fin, il annonce la mort de Dieu en disant que la couronne est donnée à l'homme (240), signalant ainsi le commencement du règne de l'homme (251).

Goetz représente l'athéisme sartrien. Il est un personnage à travers lequel Sartre se moque de l'idée de l'existence de Dieu :

... Je suppliais, je quémandais un signe, j'envoyais au Ciel des messages: pas de réponse. Le Ciel ignore jusqu'à mon nom ... A présent je connais la réponse : rien. Dieu ne me voit pas. Dieu ne m'entend pas. Dieu ne me connaît pas. Tu vois ce vide au-dessus de nos têtes ? C'est Dieu. Tu vois cette brèche dans la porte? C'est Dieu. Tu vois ce trou dans la terre ? C'est Dieu encore. Le silence, c'est Dieu. L'absence, c'est Dieu. Dieu, c'est la solitude des hommes. Il n'y avait que moi : j'ai décidé seul du Mal ... (237-238). 
Sa comédie du bien va se terminer par un crime : il poignarde le curé Heinrich qui s'oppose à ses idées. Par cet acte, il rejoint son groupe (les héros sartriens) qui affirment souvent leur liberté par le tragique.

Caligula de Camus est comparable au héros sartrien dans la poursuite de sa liberté. Il fait manifester sa liberté d'une manière tragique en tuant et en torturant ses sujets. Pour lui, la liberté consiste à faire souffrir les autres. Il déclare un jour à ses sujets :

... il y aura famine demain. Tout le monde connait la famine, c'est un fléau ... et j'arrêterai le fléau quand il me plaira ... Après tout, je n'ai pas tellement de façons de prouver que je suis libre. On est toujours libre aux dépens de quelqu'un. C'est ennuyeux, mais c'est normal ... (66).

Son règne est marqué des actes cruels et sanguinaires. Il va jusqu'au bout dans la poursuite de sa liberté et finit par nier sa mort en criant qu'il est 'encore vivant' (150) comme Hugo qui se déclare 'non récupérable' devant ses camarades à la fin des Mains sales.

\section{Conclusion}

Ayant considéré les idées centrales de l'idéologie sartrienne vis-à-vis les comportements des personnages qu'il crée dans ses œuvres théâtrales, nous constatons qu'ils existent deux types de personnages dans le théâtre sartrien: les personnages 'typiques' et les personnages 'atypiques'. Tandis que les personnages 'typiques' conforment à travers leur comportement, à l'idéologie sartrienne, les 'atypiques' sont bouleversés de l'intérieur, par des conflits souvent causés par 
le regard d'autrui ; ce qui empêche alors la réalisation de leur liberté totale telle que la conçoit Sartre.

On constate aussi que la notion de la liberté dans l'œuvre théâtrale de Sartre est souvent tragique. On y trouve souvent les héros qui affirment leur liberté aux dépens des autres. Hugo tue Hoederer, Oreste tue Egisthe et Clytemnestre, Goetz, tue le curé Heinrich, Lucie fait étrangler François. Nous constatons alors que le vrai personnage sartrien est celui qui choisit librement une voie tout à fait différente, ce qui fait que les autres ne le comprennent pas. Il est un homme solitaire parce que la voie qu'il choisit n'est pas la voie des autres. Il se décide par lui-même sans aucun guide ou recommandation. Il est condamné à la lutte pour affirmer sa liberté, et au moment où il réussit, il est prêt à assumer la responsabilité de son choix et de son acte.

\section{Euvres Citées}

Adrian, Hoven. 'Forger des mythes : le théâtre de Sartre, un théâtre de situations - Les mouches', paru dans Loxias, mis en ligne le 15

Janvier, 2004. Consulté le 24 mai, 2010.

http://revel.unice.html.

Camus, Albert. Caligula suivi de Le malentendu. Paris : Gallimard, 1972.

Contat, Michel et al. Un théâtre de situation. Nouvelle Edition, Paris : Gallimard, 1992.

Dussane. Notes de théâtre. : Paris: Lardanchet, 1951.

Jeanson, Francis. Le problème moral et la pensée de Sartre. Paris : Seuil, 1971.

Lafarge, René. Jean-Paul Sartre: His Philosophy: Indiana, U. S. A., University of Notre Dame Press, 1970. 
Launay, Claude. Le diable et le bon Dieu - Sartre. (Profil d'une œuvre) Paris : Hatier, 1974.

Lecherbonnier, Bernard. Huis clos (profil littéraire). Paris : Hatier, 1972. Noudelmann, François. Huis clos et Les mouches de Jean-Paul Sartre. Paris: Gallimard, 1993. Sartre, Jean-Paul. Huis clos. Paris : Gallimard, 2000.

_ _ . La putain respectueuse. Paris : Gallimard, 2005.

_ _ _. Le diable et le bon Dieu. Paris : Folio, 1984.

_ _ _. Les mains sales. Paris : Gallimard, 1948.

_ _ _. Les mouches. Paris : Gallimard, 2005.

_ _ _. L'Etre et le néant: Essai d'ontologie

phénoménologique. Paris : Gallimard, 2006.

Tremblay. 'Huis clos - thèmes'. N.p, n. d, n. pag. Consulté le 15 mars, 2010. http://webperso.mrdiomqc.ca/biblio.huisclosthemes/htm.

*Dr. Chioma Faith Uzoho is of the Department of Modern European Languages, Nnamdi Azikiwe University, Awka. 